\title{
Early radiographic and clinical outcomes of minimally displaced proximal fifth metatarsal fractures: cast vs functional bandage
}

\author{
Carlo Biz ${ }^{1}$ \\ Marco Zamperetti ${ }^{1}$ \\ Alberto Gasparella ${ }^{1}$ \\ Miki Dalmau-Pastor 2,3,4 \\ Marco Corradin'1 \\ Giacomo de Guttry ${ }^{1}$ \\ Pietro Ruggieri ${ }^{1}$ \\ 1 Orthopaedic Clinic, Department of Surgery, \\ Oncology and Gastroenterology DiSCOG, \\ University of Padua, Padova, Italy \\ 2 Human Anatomy and Embryology Unit, \\ Experimental Pathology and Therapeutics \\ Department, University of Barcelona, Barcelona, \\ Spain \\ ${ }^{3}$ Faculty of Health Sciences at Manresa, University \\ of Vic Central, University of Catalonia, Manresa, \\ Spain \\ 4 GRECMIP: Groupe de Recherche et d'Etude en \\ Chirurgie Mini-Invasive du Pied, Merignac, France
}

\author{
Corresponding author: \\ Carlo Biz \\ Orthopaedic Clinic - Department of Surgery, \\ Oncology and Gastroenterology DiSCOG, \\ University of Padua \\ Via Giustiniani 2 \\ 35128 Padova, Italy \\ E-mail: carlo.biz@unipd.it
}

\section{Summary}

Background: The purpose of this non-randomized retrospective study was to investigate outcomes of minimally displaced, proximal 5MTB fractures, treated by a below-knee walking cast or a functional elasticated bandage with a support of a flat hardsoled shoe.

Methods: A consecutive patient series was divided into two groups: the cast group (CG) and the functional group (FG). The subjects were radiologically and clinically evaluated according to Mehlhorn and Lawrence-Botte classification, and AOFAS Midfoot score, respectively.

Results: 154 patients were followed up for a median of 15 months (range 12-24). There was no significant difference $(p>0.05)$ among the outcomes of each fracture pattern regarding the treatment choice.
However, an earlier return to sports was noted in the FG, while Type-3 fractures achieved the worst results.

Conclusion: Type-1 and 2 minimally displaced 5MTB proximal fractures can be successfully treated conservatively without weight-bearing restriction and without benefit of a cast with respect to a functional elasticated bandage.

Level of clinical evidence: level III retrospective comparative study.

KEY WORDS: fifth metatarsal bone, foot fractures, fractures classification, Jones fracture, stress fractures.

\section{Introduction}

Fractures of the metatarsal bones are among the most frequent injuries of the foot (> 50\%) and represent 5-6\% of all fractures seen in emergency departments ${ }^{1-5}$. Multiple classification systems, over-complicating the issue, have been introduced to distinguish the different proximal fracture types of the fifth metatarsal bone $(5 \mathrm{MTB})^{6-9}$. These are considered complicated injuries due to the peculiar blood supply of this area and the multiple anatomical structures that insert in the proximal epiphysis of the $5 \mathrm{MTB}^{10}$ (Fig. 1). Torg proposed to divide the 5MTB into four zones based on common fracture lines, and sub-classifying them into acute, delayed or non-union ${ }^{11}$. At present, the simplified three-part classification proposed by Lawrence and Botte is the most commonly used ${ }^{12}$, distinguishing between tuberosity avulsion fractures, Type-1 (Zone-1); fractures at the metaphyseal-diaphyseal junction, called Jones fractures, Type-2 (Zone-2); and shaft stress fractures Type-3 (Zone-3). However, it is not widely accepted because many fracture lines lie between these zones ${ }^{13-}$ 18. In 2012, Polzer stated that non-operative treatment is indicated for metaphyseal fractures and surgical fixation for metadiaphyseal fractures, although the exact borderline between these groups remains unclear ${ }^{19}$. More recently, in 2014, Mehlhorn et al. proposed a new radiographic classification of tuberosity avulsion fractures (Zone-1), identifying 3 fracture groups at risk of secondary displacement: fractures entering in the lateral third of the 5MTB joint, fractures occurring in the middle third, and fractures in the medial third. They further divided them into two categories: non-displaced or displaced with a fracture-step-off $>2 \mathrm{~mm}^{20}$. Although Mehlhorn et al. evaluated the risk of secondary displacement, they did not evaluate patient clinical outcomes, 
Early radiographic and clinical outcomes of minimally displaced proximal fifth metatarsal fractures: cast vs functional bandage

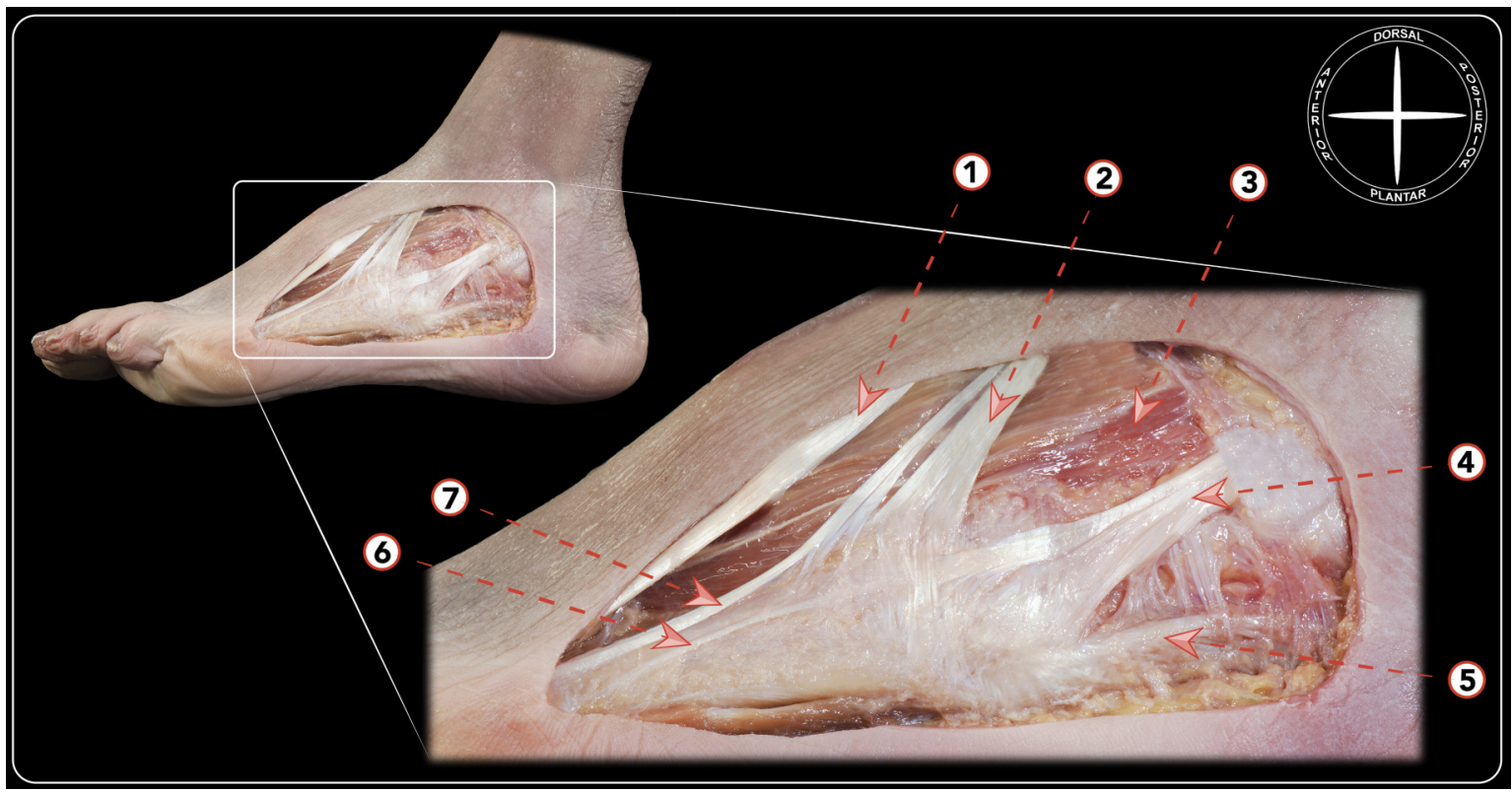

Figure 1. Dissection of the lateral side of the foot demonstrating the tendinous and aponeurotic structures inserting in the fifth metatarsal base. 1) Tendon of the extensor digitorum longus for the fourth toe. 2) Tendon of the peroneus tertius inserting in the base of the fourth and fifth metatarsal. 3) Muscle belly of the extensor digitorum brevis. 4) Tendon of the peroneus brevis inserting in the fifth metatarsal base. 5) Lateral division of the lateral component of the plantar aponeurosis inserting in the fifth metatarsal base. 6) Tendinous expansion of the peroneus tertius for the fifth toe. 7) Tendinous expansion of the peroneus brevis for the fifth toe (peroneus digiti quinti).

neither excluded from their classification the Type-2 and 3 fractures as described by Lawrence and Botte ${ }^{12}$. Management of 5MTB fractures can be challenging and is a matter of discussion in the orthopaedic community. There is little data available concerning the different fracture patterns of Zone-1, so we sought to categorize Type-1 fractures in this study to increase awareness of the typical patterns of tuberosity injuries ${ }^{19,20}$. Therefore, the purpose of this observational, retrospective, nonrandomized study, performed on a consecutive series of patients with diagnosis of acute, minimally displaced, proximal fracture of 5MTB, was to evaluate radiographic and clinical early outcomes in relation to the different fracture patterns, including sub-types-1, after conservative management without weight-bearing restriction by a below-knee walking cast or a functional elasticated bandage with the support of a flat hard-soled shoe.

\section{Material and methods}

\section{Patients}

In our study, we identified all consecutive patients who came to the attention of the Emergency Department of our Level-I Healthcare Trauma Center for acute, isolated, closed, minimally displaced fractures of the 5MTB from January 2012 to December 2014. The patients were treated without weight-bearing restriction in two different conservative methods by a below-knee walking cast or a functional elasticated bandage with the support of a flat hard-soled shoe. The choice of treatment was decided based on the preferred practice of the same orthopaedic team of 5 surgeons of our Trauma Unit, including the senior Author (C.B.). All subjects participating in this study received a thorough explanation of the risks and benefits of inclusion and gave their oral and written informed consent to publish the data. All Authors of the present study conducted this research ethically according to international standard as required by the Journal ${ }^{21}$.

\section{Inclusion and exclusion criteria}

For inclusion, all patients had to be between 18 and 65 years of age, having skeletal maturity, and having suffered an acute, isolated, closed, proximal fracture of the 5MTB with displacement of $2 \mathrm{~mm}$ or inferior and without other associated injuries. Subjects had to have a complete medical history, a foot and ankle examination, a serial radiographic evaluation, and a minimum clinical and radiographic follow-up of 12 months. Specific patient exclusion criteria were as follows: skeletally immature patients, open or inveterate fractures, multiple fractures, incomplete radiographic or chart records, history of previous foot surgery, previous ipsilateral foot surgery or fractures, tumour, lower limb deformities, rheumatologic diseases or psoriatic arthritis, diabetes, foot neuropathy and vascular insufficiency.

\section{Patient assessment}

Data collection and patient assessment were performed at our institution by two investigators (A.G. and G.d.G.), not involved in patient treatment, and analysed by a third investigator (M.C.), blinded to the type of treatment. The patients were retrospectively enrolled in the present 
case series study, and all of these patients underwent radiographic assessment at two follow-up points, while clinical evaluation was performed only at the last follow-up. Age, gender, race, affected side, body mass index (BMI) and mechanisms of the trauma were collected from the electronic database of the hospital (E-Health). The patients were distributed into two groups according to the type of treatment: the group treated by a below-knee walking cast (CG) and the group managed by functional elasticated bandaging (FG) with the support of a flat hard-soled shoe.

Both treatments began in the emergency department after the trauma. In the $C G$, the walking cast was applied by an orthopaedic surgeon of our unit with the help of two accredited casting nurses, while in the $F G$, the functional bandage was applied directly by the nursing staff. The patients of the FG group were requested to buy the orthopaedic shoe the same day or the following one. In both groups, the use of crutches was suggested, and the patients were encouraged to bear weight as soon as they could tolerate it. Both types of treatment, as well as the thromboembolic prophylaxis with Nadroparin Calcium injections, were suggested for a 4-6-week period, at the discretion of the treating surgeon.

\section{Radiographic evaluation}

Radiographic data of the 5MTB proximal fractures included standard lateral, axial, and internal oblique view radiographs of the foot taken at the time of trauma in the emergency department. The radiographs were repeated on an outpatient basis at 4- to 6-week intervals at the discretion of the treating surgeon after removing the cast or the bandage, and again at a minimum follow-up of 12 months, as proposed by the study protocol. Radiographic images were taken non-weight-bearing only at the beginning and weight- bearing at the other two followups. Diagnostic LCD CORONIS 5MP display was used as viewing monitor to analyse the radiographic images of fractures, in conventional vertical or horizontal orientation ${ }^{22}$, and fracture outcomes. Each patient's serial radiographs were reviewed by the first investigator (A.G.) to determine the site and type of fracture at the time of trauma. The examination of lateral, axial and internal oblique view radiographs at different follow-ups, showing complete bridging bone/callus formation and the absence of radiolucent lines, was used to define bone healing.

On the base of the early radiographs, the fractures were classified according to Mehlhorn and Lawrence and Botte classifications, (calling the Mehlhorn subtypes of Zone-1: a, b, c) as follows (Fig. 2):

- Type-1a: fracture involving the lateral one-third metatarsal-cuboid joint (Fig. 3);

- Type-1b: fracture involving the middle one-third metatarsal-cuboid joint (Fig. 4);

- Type-1c: fracture involving the medial one third metatarsal-cuboid joint (Fig. 5);

- Type-2: fractures at the junction between the proximal diaphysis and metaphysis of the fifth metatarsal (Jones fractures) (Fig. 6 left);

- Type-3: fractures of the fifth metatarsal shaft (shaft stress fractures) (Fig. 6 right).



Figure 2. A 3-zone anatomical classification of 5MTB proximal part used during our analysis according to Mehlhorn, Lawrence and Botte studies. Zone-1a: including the lateral one-third metatarsal-cuboid joint; Zone-1b: including the middle one-third metatarsal-cuboid joint; Zone-1c: including the medial one third metatarsal-cuboid joint. Zone-2: including the junction between the proximal diaphysis and metaphysis of the fifth metatarsal. Zone-3: including the fifth metatarsal shaft. 
Early radiographic and clinical outcomes of minimally displaced proximal fifth metatarsal fractures: cast vs functional bandage

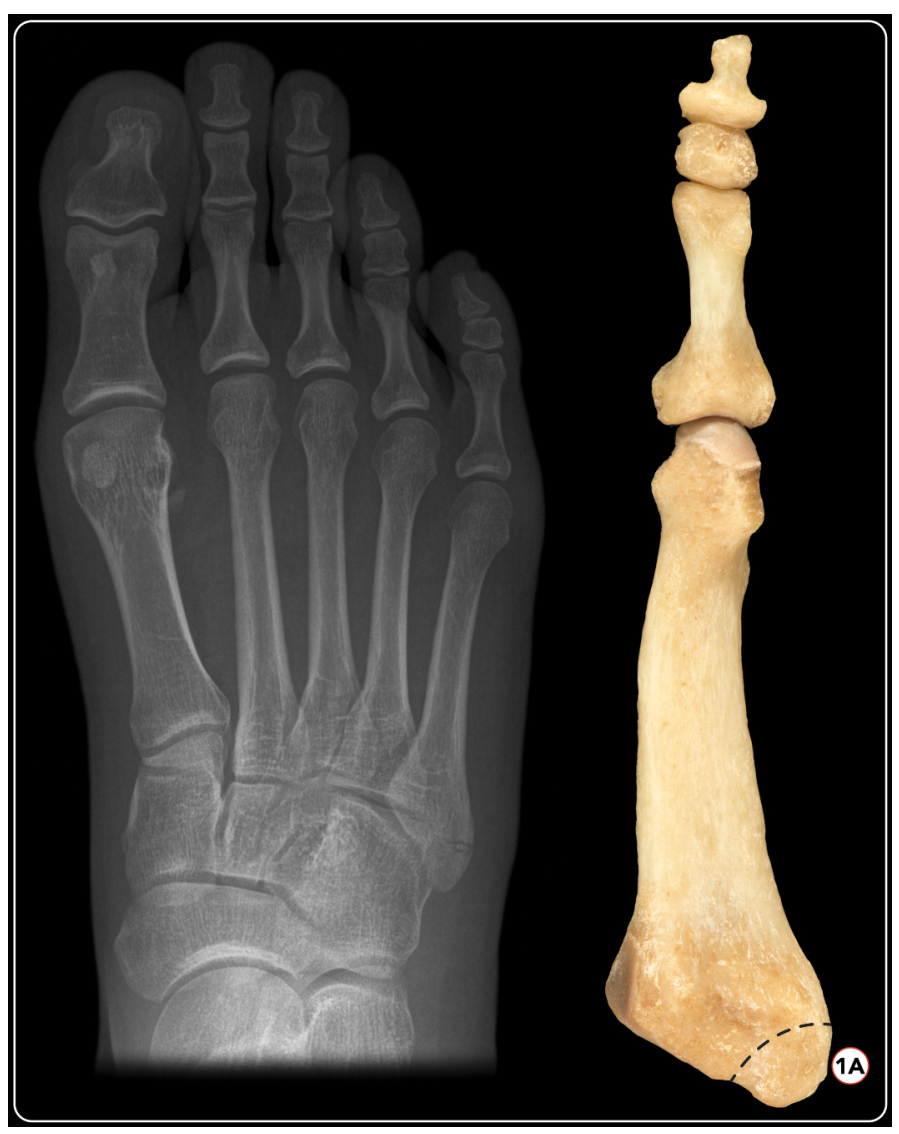

Figure 3. X-ray of a Type-1a fracture and correlation in bone model: fracture involving the lateral one-third metatarsal-cuboid joint.

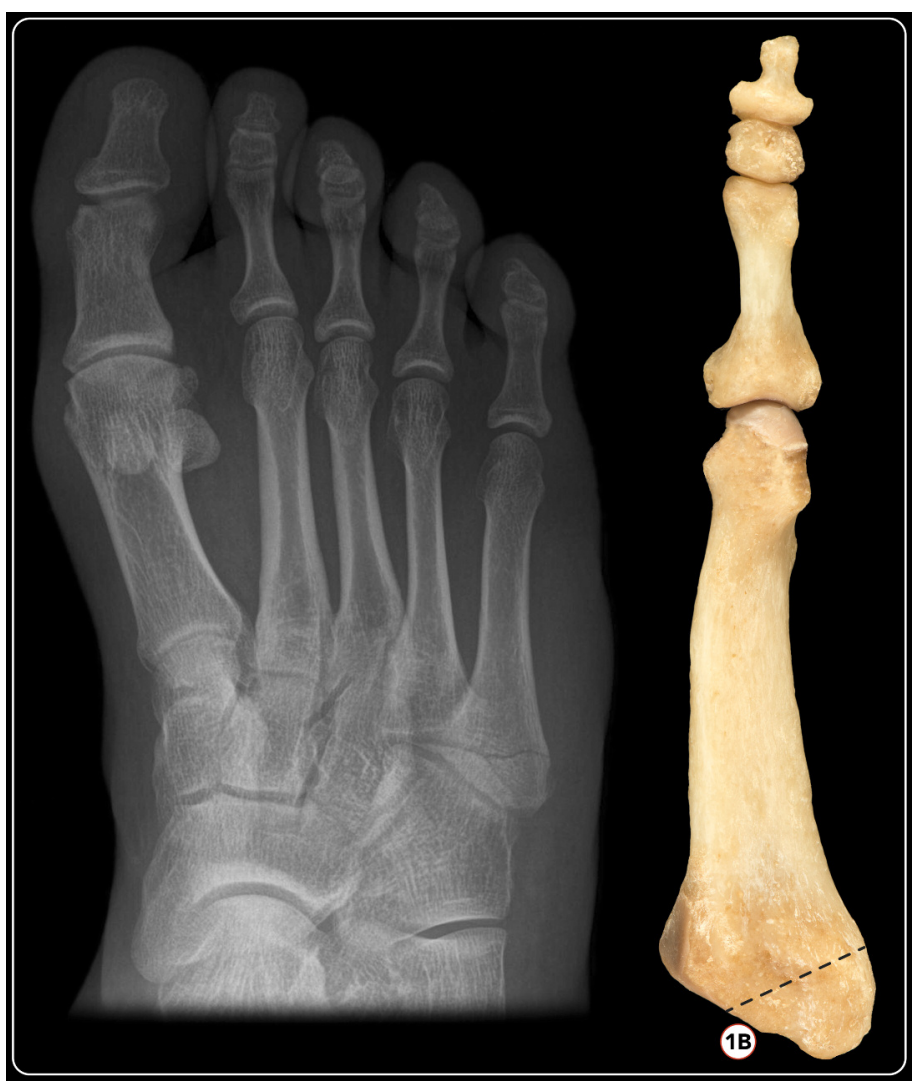

Figure 4. X-ray of a Type- $1 \mathrm{~b}$ fracture and correlation in bone model: fracture involving the middle one-third metatarsal-cuboid joint. 




Figure 5. X-ray of a Type-1c fracture and correlation in bone model: fracture involving the medial one third metatarsal-cuboid joint.

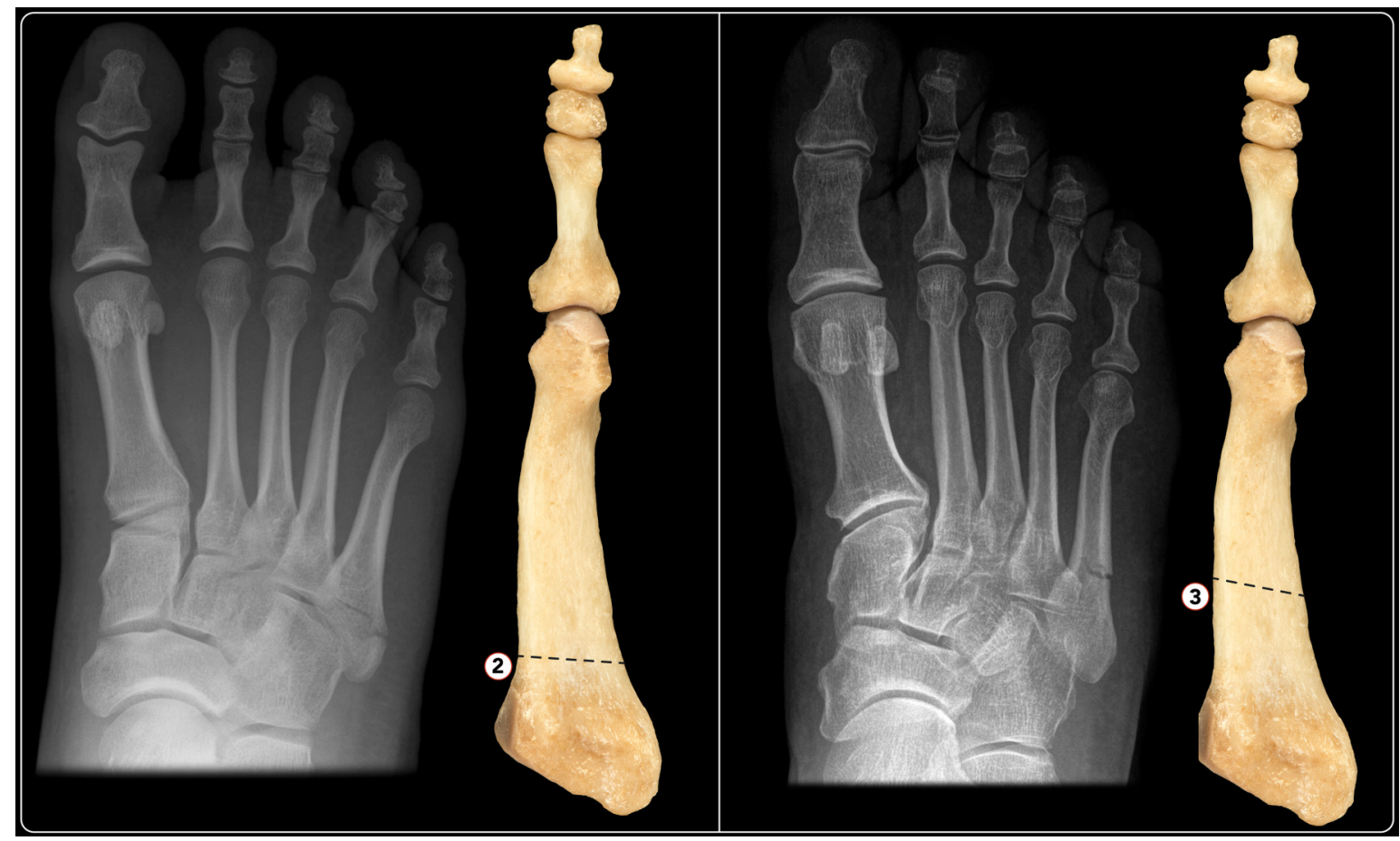

Figure 6. X-rays of Type-2 and Type-3 fractures and correlation in bone models: Type-2 (left): fractures at the junction between the proximal diaphysis and metaphysis of the fifth metatarsal (Jones fractures); Type-3 (right): fractures of the fifth metatarsal shaft (shaft stress fractures). 
Early radiographic and clinical outcomes of minimally displaced proximal fifth metatarsal fractures: cast vs functional bandage

\section{Clinical evaluation}

First, clinical information, included complications, after early evaluation at a 4- to 6-week period was obtained for each participant. Second, at the time of this study, a phone contact was attempted for all patients, and a follow-up appointment was fixed. Patients who returned were examined by the second investigator (G.d.G), and clinical results were measured using the AOFAS Midfoot score ${ }^{1}$. Clinical healing was defined as the absence of pinpoint tenderness on fracture site palpation and the patient's ability to bear weight on the involved foot without discomfort. Further, the following parameters were assessed: sport type and activity level (high level athletes training more than 14 hours per week; recreational athletes less than 14 hours per week), time until return to full weight bearing, to work and to sport activities.

\section{Statistical analysis}

Statistical analyses were performed by an independent statistician (A.C.F) from the Department of Statistics of our university using SAS 9.2 (SAS Institute Inc., Cary, NC, USA) for Windows. The comparison of the patients' demographic, anthropometric and clinical characteristics between the two treatment groups was performed with the unpaired t-test for quantitative variables. Statistical significance was set at $p<0.05$.

\section{Results}

\section{Patients' demographic data}

During a 3-year period, 188 patients with the same number of proximal fractures of 5MTB meeting the inclusion criteria were evaluated. We could not evaluate 34 patients as 13 refused to participate, and 2 were dead at time of analysis; a follow-up address could not be retrieved for 19 subjects. Of 154 patients, 74 were treated by immobilisation in a below-knee cast (CG) and 80 with a functional elasticated bandage (FG) and the support of a flat-soled orthopaedic shoe. Resulting patient data are shown in Table I. There were 94 women (61\%) and 60 men (39\%), all Caucasian subjects. The mean patient age at the time of injury was 43 years (range 15-65) for all fracture types (38 yrs for Type-1a, 45 yrs for Type-1b, 41 yrs for Type-1c, 45 yrs for Type-2 and 57 yrs for Type-3), with no significant difference between groups. The right side was involved $74(48 \%)$ times and the left 80 (52\%) times. Mean BMI was $26.3 \mathrm{~kg} / \mathrm{m}^{2}$ (range 20-29) for all fracture types (24 for Type-1a, 23 for Type-1b, 26 for Type-1c and Type-2, and 27 for Type-3), and 55 (36\%) smoked more than 20 cigarettes/day. Fifty-two patients (34\%) were recreational athletes, and 102 (66\%) did not practice any sports. None of our patients was a professional athlete. Mean follow-up time was 15

Table I. Patients' parameters, distinguished according to the 3-zone classification system used (as proposed by Mehlhorn, Lawrence and Botte), for each group of patients treated by below-knee walking cast (CG) or functional elasticated bandage (FG).

PATIENTS' PARAMETERS

\begin{tabular}{|c|c|c|c|c|c|}
\hline NUMBER & $1 \mathrm{a}$ & $1 \mathrm{~b}$ & $1 c$ & 2 & 3 \\
\hline Cast Group (CG): & 15 & 14 & 14 & 25 & 6 \\
\hline Functional Group (FG): 80 & 20 & 20 & 19 & 17 & 4 \\
\hline \multicolumn{6}{|c|}{ AOFAS MIDFOOT SCORE (100 points) } \\
\hline Cast Group (CG) & 92.9 & 94.3 & 95 & 97.5 & 85 \\
\hline Functional Group (FG) & 95.6 & 97.5 & 96 & 92.5 & 83 \\
\hline \multicolumn{6}{|c|}{ SIGNS OF CONSOLIDATION (\%) } \\
\hline Cast Group (CG) & 60 & 64.3 & 64.3 & 68 & 50 \\
\hline Functional Group (FG) & 60 & 65 & 65 & 65 & 50 \\
\hline \multicolumn{6}{|l|}{ RETURN TO WORK (weeks) } \\
\hline Cast Group (CG) & 7.1 & 7 & 7.5 & 6.7 & 8 \\
\hline Functional Group (FG) & 7 & 6 & 7 & 6.8 & 8.5 \\
\hline \multicolumn{6}{|l|}{ RETURN TO SPORT (weeks) } \\
\hline Cast Group (CG) & 12 & 10 & 12.5 & 12.1 & 20 \\
\hline Functional Group (FG) & 9 & 8 & 10 & 9.4 & 22 \\
\hline
\end{tabular}

FRACTURE TYPES 
months (range 12-24). The mechanism of injury was ankle inversion sprain and adduction of the foot for most patients (108 pts, 70\%) due to indirect trauma during fall from a curb or stairs. Twenty-eight patients $(18 \%)$ reported a direct blunt trauma and $18(12 \%)$ a mixed trauma (direct blunt trauma associated to sprain). The injuries occurred during free time (72\%), sports activities (14\%), as a result of motorcycle accidents $(6 \%)$, and in a few cases, at work (8\%).

\section{Radiographic outcomes}

There were 35 Type-1a fractures, 34 Type-1b, 33 Type-1c, 42 Type-2, and 10 Type-3. Signs of consolidation were not statistically different in the two groups for each type of fracture at 4- to 6-week follow-up. Type-3 fractures showed the least consolidation (50 vs $63.7 \%, p<0.001$ ) compared to the other cases. There was no case of secondary displacement except in Type-3 fractures where there were 2 cases (one treated with cast and one with bandage), which required 8 weeks to heal, a longer time than expected.

\section{Clinical outcomes}

The clinically mean AOFAS scores at medium followup of 15 months were not significantly different among all fracture Types, treated with cast or functionally. However, the Type-3 fracture outcomes were significantly lower compared to the other types (84.2 vs 94.2, $p<0.001)$. Return to work was faster for patients of $F G$ with Type-1A fractures and longer for those of CG with Type-3 fractures. Return to work was similar in both groups $(p>0.05)$ and slightly lower in the $F G$ compared to $C G$ (8.2 vs 6.75 weeks). However, this was not statistically significant $(p=0.08)$. Return to sport was evaluated only in patients that did at least 6 hours of sport activities per week (52/154 patients). Return to sport was earlier in the $F G$ for subjects with Type- $1 b$ and $1 c$, and longer for those with Type-3. It was significantly earlier in the $F G$ than in the $C G(\mathrm{p}<$ $0.05)$, and overall, it was also significantly longer for patients with Type- 3 fractures than for those with one of the other injuries (21.2 vs 10.2 weeks).

\section{Complication rate}

There was no case of secondary displacement except in Type-3 fractures in which there were 2 cases (mentioned above) that required longer time than expected to obtain fracture healing ( 8 weeks). No other complications were found in our patient series, no refractures observed, and there were no cases of thrombo-embolism in the lower limbs. There were no cutaneous or deep infections, neither avascular necrosis of the 5MTB. However, $16 \%$ of patients of CG experienced intolerance of the cast; however, the treatment did not require change until the programmed outpatient visit.

\section{Discussion}

Although acute proximal fractures of the 5MTB are among the most frequent skeletal injuries of the foot, in the contemporary literature several studies have shown the difficulty of their treatment and consequent healing problems because of a lack of a standardized classification system and a specific management protocol ${ }^{6,7,16,17,23,24}$. For these reasons, Mehlhorn et al. defined a fracture step-off larger than $2 \mathrm{~mm}$ as a risk factor of post-traumatic non-union for Type-1 fractures, over which, several surgeons preferred an open reduction and internal fixation (ORIF) 20, 25-28. In contrast, Lawrence and Botte ${ }^{12}$ claimed that fractures of Zone-1 have excellent healing potential, thus they should be treated symptomatically in any case. Several Authors even recommended functional treatment for Type-1 and -2 fractures indifferently, both non-displaced and displaced ${ }^{29-32}$, without considering the impact of fracture dislocation, number of fragments, or involvement of the cuboid-metatarsal joint.

In agreement with recent studies ${ }^{33-35}$, the $F G$ in our cohort achieved the best results compared to the $C G$, especially for Type-1 fractures, permitting patients a more rapid return to normal activities without compromising bone union. Although there is a lack of data regarding Type-1c fractures in the literature ${ }^{20}$, we found they had a consolidation time similar to Type-2. This fracture type is reinforced by the lateral ligament complex of Lisfranc and is very stable. In these nondisplaced injuries, functional treatment seems to have had excellent results ${ }^{35}$. This is in contrast with the study of Mehlhorn, who recommended traditional ORIF for the risk of secondary displacement also for Type-1c fractures $^{20}$.

There was no significant difference in the outcomes of individual fracture patterns regarding the chosen treatment $(p>0.05)$. However, $16 \%$ of patients experienced discomfort with the cast. The preferable method is probably functional bandage with the support of a flat hard-soled shoe for 4 weeks, even if cast treatment is not contraindicated. To support our data, recently Akimau, et al. ${ }^{31}$ concluded that cast immobilisation of avulsion fractures of the 5MTB in adults provides no benefit over symptomatic treatment with elastic bandaging in shorter and longer times. Vorlat, et al. ${ }^{36}$ reported that the most significant predicting factor of a poor functional outcome of these injuries was a prolonged period of non-weight-bearing. This aspect was irrelevant in our cohort study, as weight-bearing was permitted almost immediately in both groups.

Although most Authors ${ }^{6,37}$ have reported conservative treatment outcomes of fractures involving just the tuberosity, we analysed all three zones clinically and radiographically, finding similar outcomes of fractures Type-1a, $1 b, 1 c$ and 2, without significant difference ( $p$ $>0.05)$. Based on our results, in these fractures displaced less than $2 \mathrm{~mm}$, the radiological classification used in this study is important in order to describe the different anatomical sites, although this does not have an impact on treatment outcomes. However, it is possible that the various types of fractures described by this classification system present different outcomes for displaced fractures, for which further studies are necessary. 
In our series, Type-2 fractures yielded good results in both groups analysed. No patients required surgical treatment, and we did not have cases of non-union, independent of the treatment choice. However, conservative management in these cases is controversial and also depends on patient compliance. According to Woo et al. ${ }^{38}$, there seems to be no significant difference in the radiographic union between treatment with a leg cast for unloading and soft bandage with a tolerance load, but there is less early pain in patients treated with a leg cast. Easley et al. ${ }^{39}$ recommended initial conservative treatment, and 4-6 weeks with orthopaedic brace or plaster without weight bearing, followed by an additional 4-8 weeks of functional treatment with a weight-bearing bivalve cast. In displaced fractures, surgical treatment with intramedullary screw fixation is indicated, but if optimal compression of the fracture cannot be achieved, delayed bone healing and slower return to competition in athletes may be observed ${ }^{18}$. In particular, Jones fractures could be very dangerous in ballet dancers if not recognized, occurring in an acute or chronic form. Fortunately, in dancers, they are not as common as distal shaft fractures of the fifth metatarsal (dancer's fractures), stress fractures at the base of second and third metatarsal or lateral instability of the first metatarsophalangeal joint ${ }^{40,41}$. In any case, the time of returning to sports activity for the recreational athletes of our cohort with Type-1 and 2 fractures was similar to that reported by Saxena 42 .

Our patients with Type-3 fractures reported the worst outcomes at medium follow-up of 15 months, a delayed return to work and also to sports. Although the fractures were minimally displaced $(<2 \mathrm{~mm}), 2$ dislocations that required longer treatment period occurred. For these injuries, the majority of Authors $6,7,18,39,43$ state that nonoperative treatment leads to a significantly longer time to return to sports, with a range of failure of up to $44 \%$. They recommend surgical treatment to avoid prolonged time of cast immobilisation, high rate of displacement and poor clinical results. Although it is well documented that the gold standard treatment of Type-3 fractures is the surgical one ${ }^{18,32}$, no study compares the outcomes of shaft stress fractures after conservative versus surgical treatment. Hence, the presence of a control surgical group would be desirable. This question will be addressed in a future study.

Several potential limitations may influence our findings, mainly linked to its retrospective nature and the consequent lack of randomization. Further, the fact that the patients were treated differently according to the orthopaedic surgeons' preferences, without a prestabilised management protocol, could be another bias of the study. However, to the best of our knowledge, our study is the largest in the literature, having evaluated both functional and radiological outcomes for the different fracture patterns. It includes a small number of shaft fractures (Type-3), which were treated surgically because of displacement. Our data were collected by two independent investigators and analysed by a third, blinded to the type of treatment to reduce potential bias.
Finally, we conclude that weight-bearing restrictions and perhaps a follow-up period are not necessary, as stated by Marecek and Ferguson 44,45 , respectively, for minimally displaced Type-1(all sub-types) and Type-2 fractures. In fact, the study reveals the excellent results of these fractures managed conservatively by early loading in both groups, without any benefit of the cast with to respect to the functional elasticated bandage, although an quicker return to sports was noted in the FG. In contrast, both groups showed the worst outcomes for Type-3 fractures and slower return to work and sport activities.

\section{Conflict of interest statement}

The Authors declare that they have no conflict of interest related to the publication of this manuscript, and they have not received benefits or financial funds in support of this study.

\section{References}

1. Cakir H, Van Vliet-Koppert ST, Van Lieshout EM, De Vries MR, Van Der Elst M, Schepers T. Demographics and outcome of metatarsal fractures. Arch Orthop Trauma Surg. 2011;131: 241-245.

2. Hasselman CT, Vogt MT, Stone KL, Cauley JA, Conti SF. Foot and ankle fractures in elderly white women: incidence and risk factors. J Bone Joint Surg Am. 2003;85(5);820-824.

3. Herrer-Soto JA, Scherb M, Duffy MF, Albright JC. Fractures of the fifth metatarsal in children and adolescent. J Pediatr Orthop. 2007;27:427-431.

4. Petrisor BA, Ekrol I, Court-Brown. The epidemiology of metatarsal fractures. Foot Ankle Int. 2006;27:172-174.

5. Singer G, Cichocki M, Shalamon J, Eberl R, Höllwarth ME. A study of metatarsal fractures in children. J Bone Joint Surg Am. 2008;90:772-776.

6. Zwitser EW, Breederveld RS. Fractures of the fifth metatarsal Diagnosis and treatment. Injury. 2010;41:555-562.

7. Guettler JH, Ruskan GJ, Bytomski JR, Brown CR, Richardson JK, Moorman CT 3rd. Fifth metatarsal stress fracture in elite basketball players: Evaluation of forces acting on the fifth metatarsal. Am J Orthop. 2006;35(11):532-536.

8. Ekrol I, Court-Brown CM. Fractures of the base of the 5th metatarsal. Foot. 2004;14(2):96-98.

9. Logan AJ, Dabke H, Finlay D, N Makwana. Fifth metatarsa base fractures: A simple classification. J Foot Ankle Surg. 2007;13(1):30-34.

10. Theodorou DJ, Theodorou SJ, Kakitsubata Y, Botte MJ, Resnick D. Fractures of proximal portion of fifth metatarsa bone: anatomic and imaging evidence of a pathogenesis of avulsion of the plantar aponeurosis and the short peroneal muscle tendon. Radiology. 2003;226(3):857-865.

11. Torg JS, Balduini FC, Zelko RR. Fractures of the base of the fifth metatarsal distal to the tuberosity: Classification and guidelines for non-surgical and surgical management. JBJS. 1984;66(2):209-214.

12. Lawrence $S$, Botte MJ. Jones' fractures and related fractures of the proximal fifth metatarsal. Foot Ankle. 1993;14(6):358365.

13. Dudkiewicz I, Signh D, Blankstein A. Missed diagnosis fracture of the proximal fifth metatarsus: The role of ultrasound. Foot Ankle Surg. 2005;11(3):135-141. 
14. Fetzer GB, Wright RW. Metatarsal shaft fractures and fractures of the proximal fifth metatarsal. Clin Sports Med. 2006; 25(1):139-150.

15. Gray AC, Rooney BP, Ingram R. A prospective comparison of two treatment options for tuberosity fracture of the proximal fifth metatarsal. Foot. 2008;18(3):156-158.

16. Rosemberg GA, Sferra JJ. Treatment strategies for acute fractures and non-unions of the proximal fifth metatarsal. J Am Acad Orthop Surg. 2000;8(5):332-338.

17. Wright RW, Fischer DA, Shively RA, Heidt RS Jr, Nuber GW. Refracture of proximal fifth metatarsal (Jones) fracture after intramedullary screw fixation in athletes. Am J Sports Med. 2000;28(5):732-736.

18. Smith TO, Clark A, Hing CB. Interventions for treating proximal fifth metatarsal fractures in adults: A meta-analysis of the current evidence-base. Foot Ankle Surg. 2011;17(4):300-307.

19. Polzer H, Polzer S, Mutschler W, Prall WC. Acute fractures to the proximal fifth metatarsal bone: Development of classification and treatment recommendations based on the current evidence. Injury. 2012;43(10):1626-1632.

20. Mehlhorn AT, Zwingmann J, Hirschmüller A, Südkamp NP, Schmal H. Radiographic classification for fractures of the fifth metatarsal base. Skeletal Radiol. 2014;43(4):467-474.

21. Padulo J, Oliva F, Frizziero A, Maffulli N. Muscles, Ligaments and Tendons Journal - Basic principles and recommendations in clinical and field science research: 2016 update. MLTJ. 2016;6(1):1-5.

22. Ferran NA, Ball L, Maffulli N. Vertical or horizontal orientation of foot radiographs does not affect image interpretation. Muscles Ligaments Tendons J. 2012 Oct-Dec; 2(4):278-281.

23. Kitaoka HB, Alexander IJ, Adelaar RS, Nunley JA, Myerson MS, Sanders M. Clinical rating systems for the ankle-hindfoot, midfoot, hallux, and lesser toes. Foot Ankle Int. 1994;15(7): 349-353.

24. Mologne TS, Lundeen JM, Clapper MF, O'Brien TJ. Early screw fixation versus casting in the treatment of acute Jones fractures. Am J Sports Med. 2005;33(7):970-975.

25. Smith JW, Arnoczky SP, Hersh A. The intraosseous blood supply of the fifth metatarsal: Implications for proximal fracture healing. Foot Ankle. 1992;13(3):143-152.

26. Imre N, Kocabiyik N, Sanal HT, Uysal M, Ozan H, Yazar F. The peroneus brevis tendon at its insertion site on fifth metatarsal bone. Foot Ankle Surg. 2016;22(1):41-45.

27. Kadar A, Ankory R, Karpf R, Luger E, Elias S. Plate Fixation of Proximal Fifth Metatarsal Fracture. J Am Podiatr Med Assoc. 2015;105(5):389-394.

28. Tan EW, Cata E, Schon LC. Use of a Percutaneous Pointed Reduction Clamp Before Screw Fixation to Prevent Gapping of a Fifth Metatarsal Base Fracture: A Technique Tip. J Foot Ankle Surg. 2016;55(1):151-156.

29. Zenios M, Kim WY, Sampath J, Muddu BN. Functional treatment of acute metatarsal fractures: A prospective randomized comparison of management in a cast versus elasticated support bandage. Injury. 2005;36(7):832-835.

30. Marecek GS, Earhart JS, Croom WP, Merk BR. Treatment of Acute Jones Fractures Without Weightbearing Restriction. J Foot Ankle Surg. 2016;55(5):961-964.

31. Akimau PI, Cawthron KL, Dakin WM, Chadwick C, Blundell $\mathrm{CM}$, Davies MB. Symptomatic treatment or cast immobilisation for avulsion fractures of the base of the fifth metatarsal: A prospective, randomised, single-blinded non-inferiority controlled trial. Bone Joint J. 2016;98-B(6):806-811.

32. Giordano AR, Fallat LM. Strenght analysis of intraosseus wire fixation for avulsion fractures of the fifth metatarsal base. $J$ Foot Ankle Surg. 2004;43:225-230.

33. Lee SK, Park JS, Choy WS. Locking compression plate distal ulna hook plate as alternative fixation for fifth metatarsal base fracture. J Foot Ankle Surg. 2014;53:522-528.

34. Roche JA, Calder DF. Treatment and return to sport following a Jones fracture of the fifth metatarsal: a systematic review. Knee Surg Traumatol Arthrosc. 2013;21:1307-1315.

35. Porter DA, Dobslaw R, Duncan M. Comparison of 4.5-5.5-mm cannulated stainless steel screws for fifth metatarsal Jones fractures fixation. Foot Ank Int. 2009;30:27-33.

36. Vorlat P, Achtergael W, Haentjens P. Predictors of outcome of non-displaced fractures of the base of the fifth metatarsal. Int Orthop. 2007 Feb;31(1):5-10.

37. Shahid MK, Punwar S, Boulind C, Bannister G. Aircast walking boot and below-knee walking cast for avulsion fractures of the base of the fifth metatarsal: a comparative cohort study. Foot Ankle Int. 2013 Jan;34(1):75-79.

38. Woo Sb, Leung HB, Wong WC. Treatment of Proximal Fifth Metatarsal Fractures: Prospective Study. Hong Kong J Orthop Surg. 2004;8(2):116-122.

39. Chuckpaiwong B, Queen RM, Easley ME, Nunley JA. Distinguishing Jones and proximal diaphyseal fractures of the fifth metatarsal. Clin Orthop Relat. Res. 2008;466:1966-1970.

40. Coughlin MJ, Mann RA, Saltzman CL. Surgery of the Foot and Ankle, 8th edition. Mosby Elsevier, Philadelphia. 2007;1608: 1615-1617.

41. Biz C, Favero L, Stecco C, Aldegheri R. Hypermobility of the first ray in ballet dancer. Muscles Ligaments Tendons J. 2013 Jan 21;2(4):282-288.

42. Saxena A. Return to athletic activity after foot and ankle surgery: a preliminary report on select procedures. J Foot Ankle Surgery. 2000;39(2):114-119.

43. Fetzer GB, Wright RW. Metatarsal shaft fractures and fractures of the proximal fifth metatarsal. Clin Sports Med. 2006 Jan;25(1):139-150.

44. Marecek GS, Earhart JS, Croom WP, Merk BR. Treatment of Acute Jones Fractures Without Weightbearing Restriction. J Foot Ankle Surg. 2016 Sep-Oct;55(5):961-964.

45. Ferguson KB, McGlynn J, Jenkins P, Madeley NJ, Kumar CS, Rymaszewski $L$. Fifth metatarsal fractures: Is routine follow-up necessary? Injury. 2015;46(8):1664-1668. 